INVESTIGACIÓN ORIGINAL:

\title{
ASPECTOS BIOÉTICOS EN EL USO DE DIENTES HUMANOS COMO ESTRATEGIA PEDAGÓGICA
}

\author{
BIOETHICAL ASPECTS IN THE USE OF HUMAN TEEH AS A PEDAGOGICAL \\ STRATEGY
}

Dra. María Angélica Terreros ${ }^{1}$ PhD. Raúl Zumba Macay ${ }^{2}$ Dr. Jimmy Salazar Aarrata ${ }^{3}$ Dr. Alfredo Toala Reyes ${ }^{4}$

1 Magister en Investigación Clínica y Epidemiológica. Doctorado en Epidemiología y Salud Pública, Docente Proyectos de Titulación, Universidad Católica Santiago de Guayaquil.materrerosdehuc@gmail.com

2 Dottore Di Ricerca In Biologiae Patologia Dellinvecchiamento. Docente Farmacología General y Terapéutica, Universidad de Guayaquil. jose.zumbam@ug.edu.ec

3 Magister en Educación Superior. Docente Prótesis Removible, Universidad de Guayaquil. ¡immy.salazara@ug.edu.ec
4 Especialista en Ortodoncia. Docente Ortodoncia, Morfología, Universidad de Guayaquil. alfredo.toalar@ug.edu.ec

Correspondencia: materrerosdehuc@gmail.com

Recibido: 09-04-2021

Aceptado: 25-06-2021

Publicado: 01-07-2021

https://doi.org/10.53591/eoug.v4i2.5

\section{RESUMEN}

Aspectos éticos y legales en obtención de órganos dentales como estrategia pedagógica y en investigación, probabilidad de contaminación cruzada, medidas de bioseguridad y creación de banco de dientes. Objetivo: Analizar impacto uso de DH extraídos en el proceso educativo, procesos de desinfección/esterilización, bioseguridad y aspectos bioéticos en FPOUG. Material y Métodos.Estudio descriptivo basado en Encuesta con variables cuali - cuantitativas dirigida a 172 estudiantes de último semestre de la Facultad de Odontología de la Universidad de Guayaquil; Resultados.- uso de dientes extraídos en las asignaturas de endodoncia(28\%), operatoria dental(26\%), morfología (23\%); compra u obsequio es el tipo de adquisición de mayor frecuencia (77\%); alumno conoce que diente es portador de elementos patógenos pero (92\%) no conoce que diente adquirido proceda de persona con enfermedad infecciosa; guantes $86 \%$ o mascarilla $87 \%$ son las barreras de mayor uso; $70 \%$ realizó desinfección del diente antes de usarlo; $63 \%$ realizó proceso de limpieza y descontaminación; 41\% los conservó en algún líquido; 58\% sin líquido; $62 \%$ usa la esterilización en autoclave; $37 \%$ en formalina. Alumno considera aplicación de la Bioética en proceso quirúrgico al usar el consentimiento informado, pero la obtención de dientes solo fue a través de la compra u obsequio, no relaciona el proceso de donación al proceso de exodoncia. Conclusiones.- Es frecuente uso de dientes humanos en endodoncia, operatoria dental y morfología, su obtención es a través de compra u obsequio, conoce que diente es portador de elementos patógenos, pero no si procede de persona con enfermedad infecciosa; da importancia al consentimiento informado previa exodoncia, no se promociona proceso de donación de órganos dentarios.

Palabras clave: bioética, bioseguridad, dientes humanos, proceso pedagógico

\section{ABSTRACT}

Ethical and legal aspects in obtaining dental organs as a pedagogical and research strategy, probability of cross contamination, biosafety measures and creation of a tooth bank. Objective: Analyze the impact of the use of DH extracted in the educationaL process, disinfection/ sterilization processes, biosafety and bioethical aspect in FPOUG. Material and methods.- Descriptive study based on a survey with qualitative - quantitative variables to 172 students in the last semester of the Faculty of Dentistry of the University of Guayaquil. Results.- use of extracted teeth in endodontic courses (28\%), dentistry (26\%), morphology (23\%); purchase or gift is the type of acquisition with the highest frequency (77\%); student knows which tooth is a carrier of pathogenic elements but (92\%) does not know which acquired tooth comes from a person with infectious disease; $86 \%$ gloves or $87 \%$ mask are the most commonly used barriers; $70 \%$ performed tooth disinfection before using it; $63 \%$ carried out a cleaning and decontamination process; $41 \%$ kept them in some liquid; $58 \%$ without liquid; $62 \%$ use autoclaving; $37 \%$ in formalin. Student considers application of Bioethics in surgical process when using informed consent. But obtaining teeth was only through purchase or gift, does not relate the donation process to the extraction process. Conclusions.- The use of human teeth in endodontics, dentistry and morphology is frequent, its obtaining is through purchase or gift, knows which tooth is a carrier of pathogenic elements, but not if it comes from a person with an infectious disease; It gives importance to informed consent prior to exodontia, dental organ donation process is not promote Keywords: bioethics, biosafety, human teeth, pedagogical process 


\section{INTRODUCCIÓN}

\section{- $\quad$ Aspectos bioéticos}

Surge la Bioética como una disciplina que busca aclarar problemas éticos relacionados a la salud al hacer investigación con seres humanos, siendo las instituciones públicas responsables de la implementación de políticas de salud colectiva basadas en el emprendimiento, gestión y desarrollo de investigaciones epidemiológicas y clínicas. Vuelven una necesidad el estudio de la relevancia de los aspectos bioéticos en el uso del diente humano en el proceso pedagógico, elemento necesario en la practica preclínica de formación profesional. La literatura evidencia que en latinoamérica no se presenta una manejo adecuado en relación a la obtención de los órganos dentales, su utilidad y uso necesario para la enseñanza.

Estudios de Elaine ${ }^{1}$ refieren del poco control docente sobre la obtención de los dientes y su manejo; Paz $2019^{2}$ indica que el 98\% de los docentes tenían conocimiento sobre riesgo biológico, pero el 76,7\% no conocen el nombre específico de los agentes infecciosos; Palomer, $2016^{3}$ de la U de Chile refiere que el $68 \%$ de los docentes reconocen la importancia de los valores éticos y morales, mientras que Japón(2015) 4 manifiesta que un $45 \%$ de los estudiantes no toman ninguna medida de bioseguridad o protección. Igualmente el 58\% desconocen de normas o aspectos bioéticos y legales para obtener órganos dentales y el 69\% los llevan a sus hogares sin ningún tipo de protección y seguridad. De otra parte, Matamoros 20175 , señala un 70\% de aprobación al acto de donación de órganos, estableciendo que el rechazo a la donación esta asociada a la falta de información referente.

La necesidad de un Banco de Dientes se establece estimulando la investigación, controles en los procesos de desinfección, esterilización y almacenamiento, procesos de Consentimiento Informado de donación de órganos evitando de esta manera la contaminación cruzada y el comercio de órganos dentales. 6

La literatura científica preconiza el rigor exhaustivo en actitudes éticas y morales en el desarrollo de una investigación, su objetivo es beneficiar a la comunidad científica y general, de ahí que la información debe ser verdadera, de buena fuente, que signifique un eje para las buenas prácticas odontológicas.7,8,9

El estudiante y futuro odontólogo debe tomar decisiones éticas en relación a los trabajos de promoción, prevención, tratamiento e investigación en salud. La bioética es norma de conducta indispensable como estudiantes y como odontólogos ayudarán a nuevos trabajos investigativos en el futuro, al desarrollo de nuevas teorías, nuevos conceptos en el ámbito de la salud oral para la comunidad odontológica y para la sociedad. 10,11

Los alumnos y futuros profesionales deben estar conscientes de los principios de la Ética: autonomía del paciente, no maleficencia, beneficencia, justicia. Asociación Dental Americana 201812

\section{- Diente humano como estrategia pedagógica}

El diente humano, temporario y permanente, compuesto de tejidos mineralizados además de la masticación cumple funciones como la fonética y estética.6,13 El alumno en el proceso de enseñanza - aprendizaje utiliza el diente humano como estrategia pedagógica, siendo su modo de obtención de mucha importancia, pues el diente humano es considerado un órgano. FDI World Dental Federation, 2018,14 de ahí la necesaria consideración de aspectos éticos como considerar el registro de su origen, ${ }^{15}$ se ignora si la pieza dental ha seguido los procesos de desinfección, esterilización y alacenamiento, lo que podría ser causa de una contaminación cruzada. 16

El diente humano es una herramienta válida como en el proceso de enseñanza aprendizaje, para trasmitir el conocimiento a través de diferentes etapas, a través del ingenio, la inteligencia, la instrucción, desarrollo de habilidades. $^{20}$. La necesaria vinculación de la teoría con la práctica pasa por procesos intermedios como el diálogo, la autocrítica consciente, resultados variables. ${ }^{21}$

Hoy se menciona el uso de dientes artificiales para este aprendizado vinculante, de anatomía similar, sirven para el desarrollo de destrezas manuales, sin embargo, no presentan la textura de los dientes naturales. ${ }^{22,23}$

De ahí que lo ético sea la obtención de dientes a través de un banco de dientes, encargado del proceso de desinfección, esterilización, almacenamiento, registro de su origen, explicando a los alumnos el protocolo adecuado de la manipulación de estos órganos. ${ }^{17}$

\section{- Bioseguridad en el uso de dientes humanos.}

La aplicación de la bioseguridad tiene como objetivo disminuir la propagación de los diferentes microorganismos vinculados a accidentes por exposición de fluídos corporales y sangre, resguardando la salud de alumnos y docentes ${ }^{18}$ Se recomienda el uso de guantes, batas manga larga, gafas, mascarillas de protección para evitar contacto directo con tejidos y fluidos contaminados. ${ }^{19}$

El desconocimiento de aspectos éticos y legales en la obtención de órganos dentales, hace que el alumno los almacene en sus hogares sin ningun tipo de prevención exponiendose a una fuente de contaminación con microrganismos patógenos. ${ }^{23}$

El seguimiento de protocolos éticos adecuados en la obtención y desinfección del órgano dental, durante todo el aprendizaje permiten empoderar al alumno de una orientación real al 
momento de realizar sus prácticas, adquiere conciencia de su anatomía, morfología, estructura real del órgano dental. ${ }^{24}$

La Administración de Salud y Seguridad Ocupacional (OSHA) en Estados Unidos permite que los odontólogos entreguen los órganos dentales extraídos a sus pacientes, pero correctamente desinfectados y esterilizados. ${ }^{25}$ de no haber requerimiento del diente, pueden ser depositados en contenedores de desechos médicos especialmente marcados como desechos potencialmente infecciosos 26

Aspectos legales de la donación de órganos dentales

Los profesionales odontólogos pueden donar órganos dentales extraídos a las Facultades de Odontología para las prácticas preclínicas o de investigación. ${ }^{25}$ Otro método es la donación de órganos de manera legal y ética, es en las clínicas de la Facultad, previa explicación detallada del alumno al paciente de los fines y usos que se dará al diente, la firma de un Consentimiento informado de donación, asegurando la privacidad del paciente así como el registro de sus datos clínicos sobre enfermedades sistémicas, que podrían estar relacionados con el diente. 27

En el 2011 El Ministerio de Salud Pública del Ecuador ${ }^{28}$ en la Ley Orgánica de Donación y Trasplante de Órganos, Tejidos y Células., decreta el Art. 5.- Que tanto los receptores como los donantes tendrán los mismos derechos establecidos dentro del proceso de la donación de órgano garantizando al donador el fin para el que se realizó la donación y no otro uso.

- Consideraciones en la creación de un banco de dientes,
El uso correcto de dientes humanos para la enseñanza será óptima a través de la creación de un biobanco dental que registre el origen de los órganos y mantenga la bioseguridad necesaria, utilizada para la facilidad de enseñanza y la investigación científica. ${ }^{30}$

Será una organización sin fines de lucro, vinculada a una Institución privada o pública con el fin de ayuda dirigida a la salud, a la formación profesional y a la investigación científica. Proveída de diversos protocolos, normas y métodos para su creación, organización y reglamentos para proteger los derechos de los donantes y mantener la calidad en investigación. ${ }^{29}$

Con estos antecedentes, este estudio tiene como objetivo evaluar en los estudiantes de los últimos semestres de la Facultad Piloto de Odontología de la Universidad e Guayaquil. Aspectos relacionados el número de dientes humanos extraídos utilizados en proceso educativo y tipo de adquisición, los conocimientos sobre elementos patógenos relacionados al diente o al donador; la Bioseguridad referente a los dientes extraídos en prácticas preclínicas; la preparación del diente a utilizar, los elementos utilizados en el proceso de desinfección y los aspectos bioéticos en el proceso de la exodoncia.

\section{MATERIALES Y MÉTODOS}

Es una investigación cuali-cuantitativa, documental de diseño descriptivo, método deductivo, se realizó encuesta a 172 estudiantes del último semestre de la Facultad de Odontología sobre aspectos relacionados al uso de dientes humanos en el proceso pedagógico y los aspectos bioéticos relacionados.

\section{RESULTADOS}

1.- NÚMERO DE DIENTES HUMANOS EXTRAÍDOS UTILIZADOS EN PROCESO EDUCATIVO Y TIPO DE ADQUISICIÓN
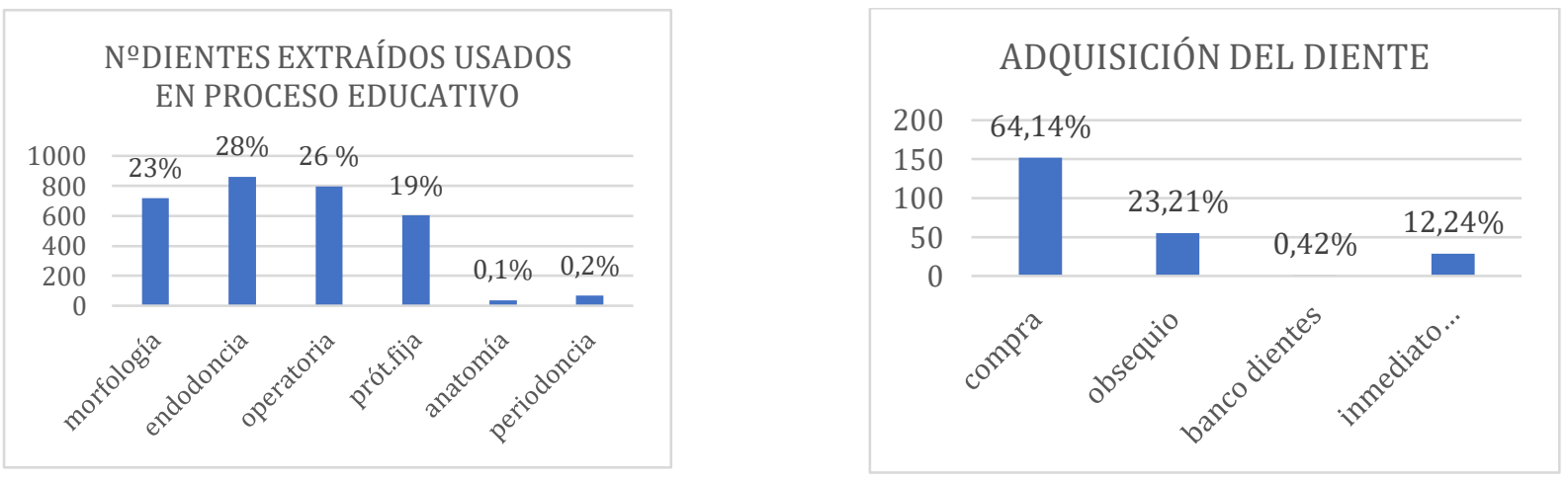

Gráfico 1. Dientes extraídos usados en el proceso pedagógico

Gráfico 2.- Adquisición de dientes para uso en proceso pedagógico. 
A la revisión de los datos se observa que la mayor frecuencia de uso de dientes extraídos en el proceso educativo se da en las asignaturas de endodoncia, operatoria dental y en morfología estableciéndose que los tipos de adquisición de mayor frecuencia son a través de la compra o el obsequio.

\section{2.-CONOCIMIENTO SOBRE ELEMENTOS PATÓGENOS RELACIONADOS AL DIENTE O AL DONADOR}

\begin{tabular}{l}
\begin{tabular}{|l|}
\hline CONOCIMIENTO \\
DIENTE/PATÓGENOS
\end{tabular} \\
\hline \\
\hline
\end{tabular}

Se establece que el alumno, en su mayoría, tiene claro conocimiento que el diente es portador de elementos patógenos, pero una alta frecuencia de estudiantes no tienen conocimiento de que el diente adquirido proceda de alguna persona con enfermedad infecciosa.

\section{3.-BIOSEGURIDAD REFERENTE A DIENTE EXTRAÍDO EN PRÁCTICAS PRECLÍNICAS}

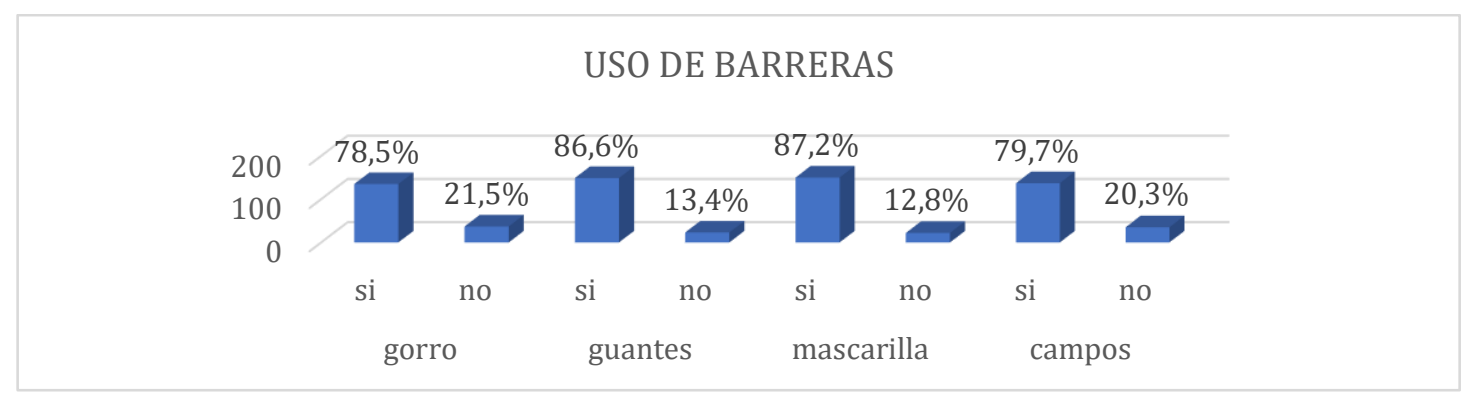

El presente estudio evidencia una alta frecuencia de uso de barreras como gorro, guantes o mascarilla y campos; es notorio que casi la cuarta parte de la población de estudio no utiliza gorro ni campos.

\section{4.-PREPARACIÓN DEL DIENTE A UTILIZAR}

\begin{tabular}{|c|c|c|c|c|c|c|c|c|c|c|c|}
\hline \multicolumn{12}{|c|}{ 5.-ACTIVIDAD PREVIA A SU PRACTICA PRECLÍNICA } \\
\hline \multicolumn{2}{|c|}{$\begin{array}{l}\text { DESINFECCIÓN } \\
\text { DEL DIENTE }\end{array}$} & \multicolumn{2}{|c|}{$\begin{array}{c}\text { PROCESO DE } \\
\text { LIMPIEZA Y } \\
\text { DESCONTAMINACIÓN }\end{array}$} & \multicolumn{2}{|c|}{$\begin{array}{l}\text { UTILIZÓ ELEMENTOS DE } \\
\text { DESINFECCIÓN/ESTERILIZACIÓN }\end{array}$} & \multicolumn{2}{|c|}{$\begin{array}{l}\text { MANTUVO } \\
\text { CONSERVADOS } \\
\text { EN ALGÚN } \\
\text { LÍQUIDO }\end{array}$} & \multicolumn{2}{|c|}{$\begin{array}{l}\text { MANTUVO } \\
\text { CONSERVADOS } \\
\text { EN CAJA SIN } \\
\text { LÍQUIDO }\end{array}$} & \multicolumn{2}{|c|}{ ESTERILIZACIÓN } \\
\hline $\mathrm{Si}$ & no & si & no & si & no & si & no & si & no & $\begin{array}{l}\text { ESTERILIZACIÓN } \\
\text { AUTOCLAVE }\end{array}$ & $\begin{array}{c}\text { ESTERILIZACIÓN } \\
\text { FORMALINA }\end{array}$ \\
\hline 121 & 51 & 109 & 63 & 92 & 80 & 72 & 100 & 103 & 69 & 108 & 66 \\
\hline $70,35 \%$ & $29,7 \%$ & $63,5 \%$ & $36,5 \%$ & $54,4 \%$ & $45,6 \%$ & $41,9 \%$ & $58,1 \%$ & $58,9 \%$ & $41,1 \%$ & $62,1 \%$ & $37,9 \%$ \\
\hline
\end{tabular}

Aunque un alto porcentaje del grupo de estudio indica que realizó desinfección del diente antes de usarlo, más de la cuarta parte de la población no lo hizo, no realizó proceso de limpieza y descontaminación, pero casi la mitad de los alumnos indica que utilizó elementos de desinfección y esterilización; menos de la mitad los tuvo conservados sin líquido, su esterilización los hizo en autoclave, menos de la mitad de los alumnos usaron la formalina. 


\section{5.- ELEMENTOS UTILIZADO EN EL PROCESO DE DESINFECCIÓN}

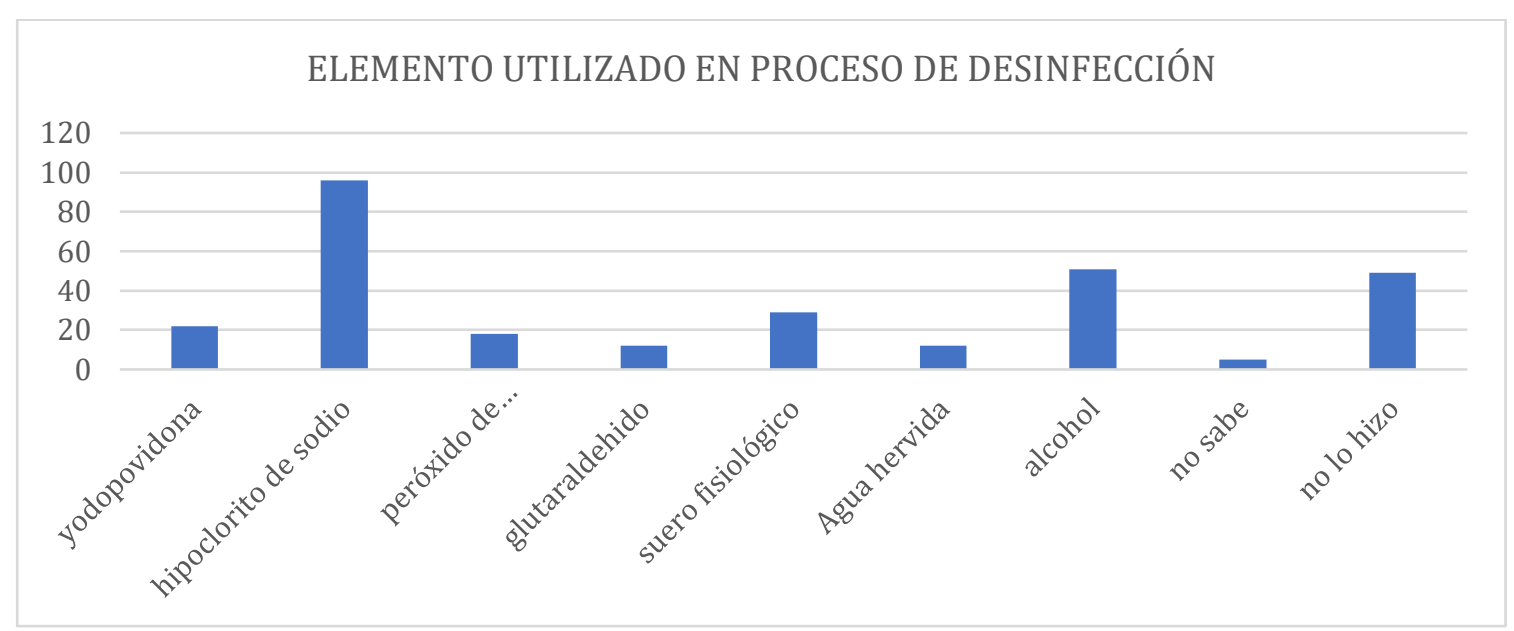

El hipoclorito de sodio es el elemento más utilizado en el proceso de desinfección seguido del alcohol y el suero fisiológico, siendo alta la frecuencia de quienes no lo hicieron.

6.-USO DE LA BIOÉTICA EN EL PROCESO DE LA EXODONCIA.

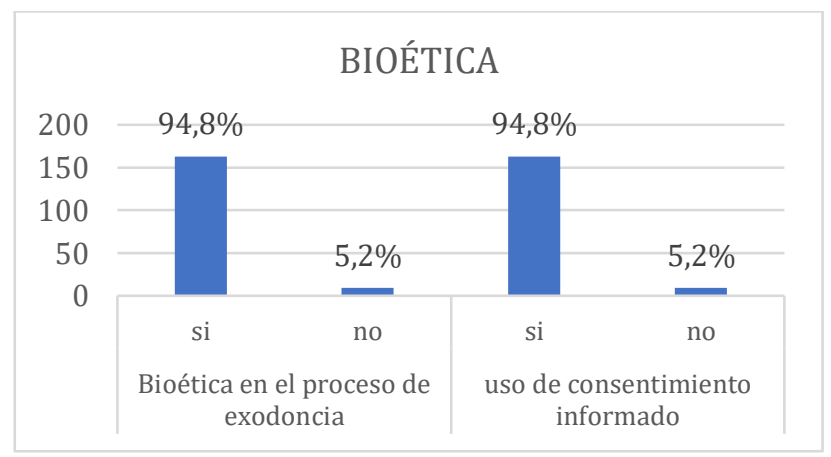

Se puede afirmar que el alumno considera que aplica los principios de la Bioética en el proceso quirúrgico aplicando el consentimiento informado, pero la obtención de sus dientes solo fue a través de la compra u obsequio, no relaciona el proceso de donación al proceso de exodoncia.

\section{DISCUSIÓN}

Los resultados de esta investigación evidencian que los alumnos limitan los aspectos bioéticos al proceso de la exodoncia cuando usan el consentimiento informado, 95\%; mientras que estudios de Japón (2015) manifiestan que 58\% de los estudiantes desconocen normas o aspectos éticos y legales para obtener órganos dentarios. Se establece asi mismo, que Matamoros 20175, relaciona el rechazo a la donación con la falta de información referente.

Se evidencia el uso del diente humano como estrategia pedagógica en las cátedras de Morfología 23\%, Endodoncia $28 \%$, Operatoria dental 26\%, Prótesis fija 19\%, en tanto, investigaciones de Japón 82015) señalan que el 100\% de los encuestados lo usan en la cátedra de Morfología y Endodoncia; siendo su modo de obtención de mucha importancia, pues el diente humano es considerado un órgano. FDI World Dental Federation, 2018,14. Se establece que el 64\% de los alumnos del presente grupo de estudio adquieren dientes para el proceso educativo a través de la compra; similares datos a la Investigación de Japón 2015, quien señala que el $71 \%$ los obtuvo con igual procedimiento. .

Minaya 2017 y Espíndola 2018, recomiendan la aplicación de la bioseguridad para disminuir la propagación de los diferentes microorganismos vinculados a accidentes por exposición de fluídos corporales y sangre, resguardando la salud de alumnos y docentes,. A este respecto el presente estudio manifiesta que el $77 \%$ de los alumnos tiene conocimiento de que el diente es portados de elementos patógenos, muy similar a los resultados de Japón 2015, 79\%; pero de otra parte mientras en este estudio se evidencia que el $92 \%$ no tiene conocimiento de alguna enfermedad infecciosa 
del donador, investigación de Japón señala que apenas el 42\% sabe que si se puede contagiar de una enfermedad infecciosa. En cuanto a bioseguridad, esta investigación señala que sobre el $87 \%$ de los alumnos del grupo de estudio usa guantes y mascarilla, Japón indica que el $59 \%$ usa barreras de bioseguridad. Para la desinfección p el $90 \%$ de los estudiantes usa el hipoclorito de sodio. mientras que Japón (2015), indica que el $56 \%$ usa métodos de desinfección y el $47 \%$ usa el glutaraldehido. La conservación de los dientes, esta investigacion señala que el $60 \%$ los mantiene a los dientes en caja sin líquido, mientras la investigación de Japón indica el $40 \%$ los mantiene en caja sin líquido.

\section{CONCLUSIONES}

- La mayor frecuencia de uso de dientes extraídos se da el proceso educativo de las asignaturas de endodoncia, operatoria dental y en morfología, con adquisición a través de la compra o el obsequio.

- El alumno, en su mayoría, tiene claro conocimiento que el diente es portador de elementos patógenos, pero no lo relaciona con alguna enfermedad infecciosa de su portador.

- Aunque evidencian uso de barreras de bioseguridad, una cuarta parte de la población de estudio no utiliza gorro ni campos.

- Casi la mitad de los alumnos realizaron proceso de limpieza y descontaminación, utilizando elementos de desinfección y esterilización; los conservaban en seco, utilizaron la autoclave para la esterilización, muy pocos usaron formalina

- El hipoclorito de sodio es el elemento más utilizado en el proceso de desinfección seguido del alcohol y el suero fisiológico, siendo alta la frecuencia de quienes no lo hicieron.

- Se puede afirmar que el alumno considera que aplica los principios de la Bioética en el proceso quirúrgico aplicando el consentimiento informado, pero no se ha establecido el proceso de donación de órgano

\section{RECOMENDACIONES}

- Se establece la necesidad de principios éticos, legales y de bioseguridad, en la obtención de órganos dentales por parte de alumnos y docentes para un óptimo desarrollo del aprendizaje teórico-práctico.

- Se establece la necesidad institucional de la creación de un biobanco dental en la Facultad, para que se facilite de forma ética y legal y con todas las normas de bioseguridad, la obtención de dientes para que estudiantes y docentes puedan utilizarlo en el aprendizaje y en la investigación.

\section{REFERENCIAS BIBLIOGRÁFICAS}

1. Elaine , F. (2014). Análisis ético de la obtención de dientes utilizados por estudiantes de una graduación en Odontología. Rev. Bioét., 22(1), 171-5.

2. Paz , M. (2019). Conocimientos, actitudes y prácticas de normas de bioseguridad y riesgo biológico en odontólogos de práctica privada de tres ciudades de Nicaragua. Odontologia Sanmarquina, 22(1), 19-25.

3. Palomer, L. (2016). Medición de los valores éticos y morales enseñados en la carrera de Odontología de la Pontificia Universidad Católica de Chile, desde la apreciación docente. 19(2), 77-84.

4. Japon , M. (julio de 2015). Aspectos legales y de bioseguridad en el uso de dientes humanos en la Cátedra de Morfología y Endodoncia en la Facultad de Odontología de la Universidad Central del Ecuador en el terceroy quinto semestre periodo octubre -marzo. 1-96. 25000/4561/1/T-UCE-0015-153.pdf

5. Matamoros, M. (2017). Conocimientos, actitudes y creencias sobre donación y trasplanteen Honduras. Rev. cienc. forenses Honduras, 3(1), 1-8.

6. Ortiz , L. (19 de 09 de 2018). Diseño del proyecto de un banco de dientes para la carrera de odontología de la ucsg. Universidad Catolica Santiago de Guayaquil, 1-38.

7. Salazar, M. (marzo de 2018). La importancia de la ética en la investigación. Universidad y Sociedad, 10(1), 305311.

8. Alvarez, P. (febrero de 2018). Ética e investigación. boletín virtual, 7(2), 122-149. https://www.academia.edu/43452514/\%C3\%89TICA_ E_INVESTIGACI\%C3\%93N_ETHICS_AND_RESEARCH_PR IMER

9. Suarez, D. (31 de 08 de 2016). Bioética, principios y dilemas éticos en Odontología. Odontología Sanmarquina de la Facultad de Odontología, 19(2), 5052.

10. Del Castillo SD, R. A. (2018). La ética de la investigación científica y su inclusión en las ciencias de la salud. Acta Med Cent, 12(2), 213-227.

11. Yañez, M. (2018). Bbioética en investigación odontológica. vision odontologica, 5(2), 95.

12. American Dental Association. (2018). The ADA Principles of Ethics and Code of Conduct. Council on Ethics, Bylaws and Judicial Affairs , 1-23. https://www.ada.org/ /media/ADA/Member\%20Cent er/Ethics/Code_Of_Ethics_Book_With_Advisory_Opinion s_Revised_to_November_2018.pdf?la=en

13. Ayala, Y. (2018). La erupción dentaria y sus factores influyentes. Correo Científico Médico, 22(4), 681-694

14. FDI world dental federation. (2018). Una visión general de las cuestiones. 1-140. file://C:/Users/USUARI 1.WIN/AppData/Local/Temp /Manual_de_EYtica_Dental_Final.pdf

15. Godani, A.. Putting the Teeth You Extracted to Good Use. Dental Reach. Recuperado el 21 de 11 de 2020, de 
https://dentalreach.today/dental-education/puttingthe-teeth-you-extracted-to-good-use/

16. Cantú, G. (12 de 2017). ¿Cómo respetar la voluntad de donar? Exigir el respeto de sí mismo. Sociedad Mexicana de Trasplantes., 6(3), 96-105. Recuperado el 10 de 10 de 2020, de https://www.medigraphic.com/cgibin/new/resumen.cgi?IDARTICULO=76379

17. Frutos , M. (2015). Ética en donación de órganos: una alianza rentable. Cuad. med. forense, 21(1), 50-56.

18. Espínola, M. (2018). Manual de Bioseguridad. Facultad de Odontología universidad nacional de asunción, 1- 51. http://proyectos.com.py/odo/wpcontent/uploads/2017/04/Manual-de-BioseguridadFOUNA.pdf

19. Minaya, Z. (2017). Manual de bioseguridad. Nuevo horizonte, 1-63. https://es.slideshare.net/helmermejiasanchez/manualde-bioseguridad-para-centro-odontolgico

20. Cabrera , B. (2016). La estrategia pedagógica como herramienta para el mejoramiento del desempeño profesional de los docentes en la Universidad Católica de Cuenca. Cubana de Educación Superior, 35(2), 72-82.

21. Hernández, I. (13 de julio de 2016). Aspectos éticos en la donación de órganos, más allá de sólo generosidad. Gastroenterol. latinoam, 27(1), 76-78.

22. Barnafi , P. (2015). percepción de estudiantes sobre la nueva metodología de simulación clínica en operatoriadental básica en la facultad de odontologíade la universidad de chile. universidad de chile facultad de odontología departamento de odontologia restauradora, 1 44. http://repositorio.uchile.cl/bitstream/handle/2250/14 1481/Percepci\%C3\%B3n-de-estudiantes-sobre-lanueva-metodolog\%C3\%ADa-de-simulaci\%C3\%B3ncl\%C3\%ADnica.pdf?sequence $=1$ \&isAllowed $=y$

23. Al-Sudani, D. (2016). Students' perceptions of preclinical endodontic training with artificial teeth compared to extracted human teeth. European Journal of Dental Education, 21(4), 72-75. doi/full/10.1111/eje.12223

24. Nawrocka, A. (2019). Extracted human teeth and their utility in dental research. Recommendations on proper preservation: A literature review. Dent Med Probl., 56(2), 185-190.

25. Raga, S. (18 de 12 de 2015). What Happens to Your Tooth After It Gets Extracted? Mental Floss. https://www.mentalfloss.com/authors/64499/Suzann e-Raga
26. Tiol-Carrillo, A. (2018). Manejo de residuos peligrosos en el consultorio dental. Revista Odontológica Mexicana, 22(3), 126-127.

27. Tello, I. (2017). Actitudes y conocimientos frente a la donación de órganos y tejidos. CASUS., 2(2), 82-88.

28. Ministerio de Salud Pública del Ecuador. (2011). Ley Orgánica de Donación y Trasplante de Órganos, Tejidos y Células. NDOT.

29. Serrano, N. (06 de 2016). Consentimiento Informado para Biobancos: Un debate ético abierto. Revista de la Universidad Industrial de Santander, 48(2), 246-256.

30. Herrera, T. (octubre de 2016). Creación de un banco de órganos dentales humanos para el aprovechamiento académico en la carrera de odontología de la universidad regional autónoma de los andes y sus protocolos de manejo. Universidad Regional Autónoma de los Andes“Uniandes"Facultad de Ciencias mdicascarrera de Odontología.

http://dspace.uniandes.edu.ec/bitstream/123456789/ 5432/1/PIUAODONT015-2016.pdf

\section{CONFLICTOS DE INTERESES}

Los autores no refieren conflictos de intereses. 